\title{
Flexible recruitment of semantic richness: context modulates body-object interaction effects in lexical-semantic processing
}

\section{Cody Tousignant and Penny M. Pexman*}

Department of Psychology, University of Calgary, Calgary, AB, Canada

\section{Edited by:}

Paul D. Siakaluk, University of Northern British Columbia, Canada

\section{Reviewed by:}

Milena Rabovsky,

Humboldt-Universität zu Berlin Germany

Ron Borowsky, University of Saskatchewan, Canada

*Correspondence:

Penny M. Pexman, Department of Psychology, University of Calgary, 2500 University Drive NW, Calgary, AB T2N 1N4, Canada. e-mail: pexman@ucalgary.ca

\begin{abstract}
Body-object interaction (BOI) is a semantic richness variable that measures the perceived ease with which the human body can physically interact with a word's referent. Lexical and semantic processing is facilitated when words are associated with relatively more bodily experience. To date, $\mathrm{BO}$ effects have only been examined in the context of one semantic categorization task (SCT; is it imageable?). It has been argued that semantic processing is dynamic and can be modulated by context. We examined these influences by testing how task knowledge modulated $\mathrm{BO}$ effects. Participants discriminated between the same sets of entity (high- and low-BOI) and action words in each of four SCTs. Task framing was manipulated: participants were told about one (is it an action? vs. is it an entity?) or both (action or entity? vs. entity or action?) categories of words in the decision task. Facilitatory BOI effects were only observed when participants knew that "entity" was part of the decision category. That BOI information was only useful when participants had expectations that entity words would be presented suggests a strong role for the decision context in lexical-semantic processing, and supports a dynamic view of conceptual knowledge.
\end{abstract}

Keywords: semantic richness, semantic categorization task, body-object interaction, lexical-semantic, task effects
The study of semantic richness effects has provided valuable insight into the process by which meaning is derived from words. The fact that lexical-semantic processing is facilitated when words have relatively more semantic neighbors (Buchanan et al., 2001), or relatively more features (Pexman et al., 2002, 2003; Grondin et al., 2009), or evoke more imagery (Balota et al., 2004), or more bodily experience (Siakaluk et al., 2008a,b; Tillotson et al., 2008) suggests that these dimensions are all relevant to semantic processing and, presumably, to semantic representation.

For instance, recent research by Siakaluk and colleagues has examined how sensorimotor experience is relevant to lexicalsemantic processing. More specifically, as a counterpoint to semantic richness variables such as imageability which focus on sensory experience, Siakaluk and colleagues were interested in the extent to which subjects' motor interactions with a word's referent affected lexical-semantic processing. To this end, Siakaluk et al. (2008a) collected body-object interaction (BOI) ratings for a series of words by having participants rate how easily they could interact with each word's referent. They then presented participants with low-BOI words (i.e., referents were relatively hard to interact with, e.g., ship) or high-BOI words (i.e., referents were relatively easy to interact with, e.g., belt) in a lexical decision task (LDT) as well as SCT with the "is it imageable?" decision category (Siakaluk et al., 2008b; Wellsby et al., 2011). In both tasks, high-BOI words were classified faster than low-BOI words. These BOI effects have been interpreted in the same framework as many other richness effects (e.g., imageability, number of features, semantic neighborhood density). That is, richer concepts generate stronger semantic activation, facilitating SCT performance, and also providing stronger feedback to orthographic units, facilitating LDT performance.

A lingering theoretical question about BOI and other richness effects, however, is whether these dimensions are a function of stable or dynamic semantic representations. Traditionally, the representation of conceptual knowledge has been characterized as stable and invariant (e.g., Collins and Loftus, 1975; Fodor, 1975). That is, the process of generating word meaning involves activation of a fixed set of properties or features and this process is not modified by task demands. Alternatively, it has been argued that the process of generating word meaning is at least to some degree context dependent (e.g., Barsalou, 1982), such that context determines the particular features activated (Hoenig et al., 2008; Kiefer and Pulvermüller, 2012). This distinction is present in more recent theories, with some proposing stable conceptual representations (e.g., Caramazza and Mahon, 2003) and others proposing more flexibility (e.g., Barsalou, 2008). The goal of the present study was to investigate whether task context modulates the effect of semantic richness, in order to establish how flexibly this information is used.

There is some evidence from previous studies that semantic richness effects vary across tasks. When several different semantic richness effects were compared across LDT and SCT, Pexman et al. (2008) reported that while the effects of contextual dispersion and number of features were significant in both tasks, the effect of number of semantic neighbors was significant only in LDT. Similarly, Yap et al. (2011) compared semantic richness 
effects across naming, LDT, and SCT, and reported that, while some richness effects were observed across all tasks (e.g., contextual dispersion, number of features), semantic neighborhood density was significant only in LDT. Based on their findings, these authors have argued that readers can dynamically adjust the kinds of information they use to suit the specific demands of the tasks that they face; depending upon a reader's goals they might emphasize some dimensions at the expense of others.

Other studies have compared richness effects within the SCT, by contrasting the effects observed under different decision categories. Pexman et al. (2003) examined how the breadth of a decision category affected categorization latencies for a critical set of items in SCT. Participants categorized low- and high-number of features birds, as well as fillers, in one of three decision categories. The categories ranged from specific to broad: is it a bird? Is it a living thing? Is it concrete? Number of features effects were observed in all three tasks, reflecting faster categorization latencies for the high- versus low-number of features birds, but the size of the number of features effect was approximately twice as large when the decision category was broad (concrete vs. abstract) than when it was narrow (bird vs. nonbird). In a similar vein, semantic ambiguity effects were observed for broad, but not narrow, decision contexts in a SCT (Hino et al., 2006). Hino and colleagues argued that with a narrow decision category participant make their decision by checking a small number of candidate features for each word, whereas with a broad decision category participants invoke more analytic processing to evaluate all activated features for each word. For example, when deciding whether a word refers to a bird, participants can focus on diagnostic semantic information such as whether the referent has feathers or a beak, while ignoring irrelevant semantic information such as how imageable the referent may be. This is not possible for more general decision contexts, where a wide array of semantic features is relevant to the category judgment.

Relatedly, Hargreaves et al. (2012) recently used fMRI to compare the neural correlates of two SCT conditions. The two SCTs involved different decision categories: is it an animal? vs. is it a concrete thing? Participants completed both tasks and, across participants, the same core set of items were presented in both tasks. The fMRI results showed relatively more activity for the animal SCT in cortical regions that have been linked to general knowledge (e.g., left superior and middle temporal gyri) while the concrete SCT showed relatively more activity in motor regions. These results are interpreted as evidence of top-down modulation of semantic processing; participants make adjustments to optimize performance in a given task and these adjustments have consequences for the activation observed.
The present experiment provides a conceptual extension of recent work examining the role of the decision context in modulating effects of semantic richness (Pexman et al., 2003; Hino et al., 2006), but here we used a more fine-grained manipulation of task context than has been achieved in previous studies. In the previous studies examining decision context effects in SCT, different items (fillers) have been included in each condition. That is, while the analyses have focused on a core set of items presented in every condition, the other items in each condition differed. Thus, the decision context was not the only thing that varied across conditions. In the present study, however, we used exactly the same item sets in every condition. The only thing that varied was the way the decision was presented to participants. Here the context manipulation was not of category breadth but rather category framing. In each of four different decision contexts, participants discriminated between the same sets of object words (concerned that the term "object" would be interpreted too narrowly we referred to these as "entity" words, and they included both lowand high-BOI words), and action words. The BOI dimension is particularly well-suited to this framing manipulation because it is relevant to a certain class of words: concrete nouns. We expected that in general high-BOI words would be classified more quickly and accurately than low-BOI words. The decision context was manipulated by varying the information participants were given about the types of items in the decision task (see Table 1).

We manipulated whether participants were told about one (i.e., is it an entity? vs. is it an action?) or both (entity or action? vs. action or entity?) categories of words present in the task. This allowed us to assess whether BOI effects depend on participants knowing that object information will be relevant to the task. If BOI effects are ubiquitous to semantic processing, then this manipulation should have no effect, and a BOI effect should be observed in every version of the decision category. If, on the other hand, semantic processing is modulated by participants' expectations about the relevant information in a task, then BOI effects should not be observed in every version of the decision category and, in particular, may be attenuated in the "is it an action?" version of the decision, where participants are not told in advance that object words will be presented for categorization.

\section{METHOD \\ PARTICIPANTS}

One hundred and fifty-nine University of Calgary undergraduate psychology students were randomly assigned to the entity ( $n=$ $41)$, entity-action $(n=39)$, action-entity $(n=39)$, or action $(n=40)$ decision conditions and participated in exchange for course credit. All participants had normal or corrected to normal vision and were fluent English speakers.

Table 1 | Study design.

\begin{tabular}{|c|c|c|c|c|}
\hline Instruction condition & $n$ & Participants' expectations & Left response button & Right response button \\
\hline Is it an entity? & 41 & Entity words and non-entity words & Low- and high-BOI words & Action words \\
\hline Is it an entity or an action? & 39 & Entity words and action words & Low- and high-BOI words & Action words \\
\hline Is it an action or an entity? & 39 & Action words and entity words & Action words & Low- and high-BOI words \\
\hline Is it an action? & 40 & Action words and non-action words & Action words & Low- and high-BOI words \\
\hline
\end{tabular}




\section{STIMULI}

The BOI ratings collected by Tillotson et al. (2008) were used to compile lists of potential low- and high-BOI entity words. In addition, a list of potential action words (e.g., jump) was selected from the MRC database (Wilson, 1988).

To ensure that the entity words were conceptually distinct from the action words, a separate group of 45 participants used a sixpoint Likert scale to rate how action-like the potential stimuli were ( $1=$ entity, $6=$ action). This information was used to compile a final list of 35 low- and 35 high-BOI words which had all received low ratings on the entity-action scale, and were matched on a number of dimensions (see Table 2). Finally, 70 action words that received high ratings on the entity-action scale were selected. All items are listed in the Appendix.

\section{PROCEDURE}

Stimuli were presented using E-Prime software (Schneider et al., 2002) on a $20^{\prime \prime}$ CRT monitor. On each trial, a $500 \mathrm{~ms}$ fixation cross was presented, followed by a $60 \mathrm{~ms}$ blank screen. This was followed by a target word. In the entity condition, participants were asked to press the left button on a response pad for words that referred to entities and to press the right button for words that referred to non-entities. In the entity-action condition, participants were asked to press the left button in response to words that referred to entities and use the right button to respond to words that referred to actions, whereas in the action-entity condition, participants responded with the left button for words that referred to actions and the right button for words that referred to entities. Finally, in the action condition, participants were asked to provide a left button response to words that referred to actions and a right button response to words that referred to non-actions. The decision category (i.e., "Is it an Entity?" "Entity or Action?" "Action or Entity?" or "Is it an Action?") was presented above each target word. Participants were asked to respond as quickly and as accurately as possible.
To ensure that participants understood the instructions, the experimenter remained in the room while six practice trials were presented. Once the practice trials were completed, the experimenter left the room and the participants completed the remaining 140 experimental trials.

\section{RESULTS}

The data for one participant from the action condition and three participants from the entity condition were removed from the final analysis due to poor categorization performance for the critical words (accuracy $<70 \%$ ). The final group sizes were thus 38 in the entity condition and 39 in the remaining three conditions.

The data for any item for which participants demonstrated less than $70 \%$ categorization accuracy were removed from the analysis: fog, back, well, song, and case (all low-BOI items) in the entity condition (3.57\% of the data), flea (low BOI), pat, and sue (both actions) in the entity-action condition $(2.15 \%$ of the data), flea (low BOI) in the action-entity condition $(0.07 \%$ of the data), and boot (high BOI) and flea (low BOI) in the action condition (1.43\% of the data). In addition, trials with response latencies faster than $350 \mathrm{~ms}$ or slower than $2500 \mathrm{~ms}$ were removed from the analysis (entity condition: $0.34 \%$ of the data; entity-action condition: $3.15 \%$ of the data; action-entity condition: $3.90 \%$ of the data; action condition: $1.55 \%$ of the data).

Mean RTs and accuracy (see Table 3) were analyzed with four (decision category: entity, entity-action, action-entity, action) $\times 2$ (BOI: low, high) mixed factors ANOVAs by subjects $\left(F_{1}\right)$ and by items $\left(F_{2}\right)$. In the subject analysis, condition was the betweensubjects variable and BOI was the within-subjects variable. In the item analysis, condition was the within-item variable and BOI was the between-item variable.

\section{RT ANALYSIS}

In the analysis of RT data, there was an interaction of decision category and BOI, $F_{1}(3,151)=10.27, p<0.001, \eta^{2}=$

Table 2 | Mean (SD) characteristics of low-BOI and high-BOI word stimuli.

\begin{tabular}{llll}
\hline Characteristic & Low BOI & High BOI & $\boldsymbol{p}$ \\
\hline BOI rating & $3.39(0.55)$ & $5.67(0.46)$ & $<0.001$ \\
Entity-action rating & $1.69(0.30)$ & $1.60(0.25)$ & 0.20 \\
Word length & $4.14(0.84)$ & $4.14(0.84)$ & 1.00 \\
Familiarity & $5.32(0.43)$ & $5.32(1.03)$ & 0.99 \\
Imageability & $5.72(0.39)$ & $5.69(0.30)$ & 0.75 \\
Concreteness & $5.67(0.38)$ & $5.76(0.34)$ & 0.33 \\
Orthographic neighbors & $9.08(5.54)$ & $87.08(6.00)$ & 0.91 \\
Kucera-Francis frequency & $96.31(219.79)$ & $95.41(202.19)$ & 0.93 \\
CELEX frequency & $116.08(303.82)$ & $53.59(6.02)$ & 0.73 \\
Standard frequency index & $52.74(8.92)$ & $1678.30(859.20)$ & 0.64 \\
Bigram frequency & $1841.62(926.46)$ & $0.71(0.14)$ & 0.36 \\
Contextual dispersion & $0.68(0.15)$ & 0.43 \\
\hline
\end{tabular}

Note: BOI ratings were taken from Tillotson et al. (2008) norms. Entity Ratings ranged from 1 (entity) - 6 (action) and were collected in a pilot study. Familiarity, imageability and concreteness measures were taken from the MRC Psycholinguistic Database [Wilson (1988)], Orthographic neighbors and bigram frequency measures were taken from the English Lexicon Project [Balota et al. (2007)]. Kucera-Francis Frequency [Kucera and Francis (1967)]. CELEX Frequency = Dutch

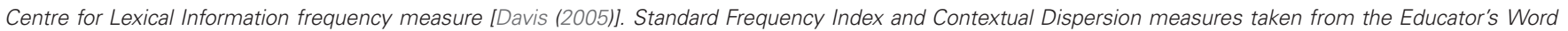
Frequency Guide [Zeno et al. (1995)]. 
Table 3 | Mean (SD) RT and accuracy for low-BOI words, high-BOI words, and action words.

\begin{tabular}{|c|c|c|c|c|c|c|c|c|}
\hline Decision category & \multicolumn{2}{|c|}{ Low BOI words } & \multicolumn{2}{|c|}{ High BOI words } & \multicolumn{2}{|c|}{ BOI effect } & \multicolumn{2}{|c|}{ Action words } \\
\hline Entity-action & $968(202)$ & $0.93(0.09)$ & $911(169)$ & $0.93(0.07)$ & -57 & 0.005 & $874(136)$ & $0.92(0.06)$ \\
\hline Action-entity & $1046(182)$ & $0.95(0.05)$ & $995(171)$ & $0.95(0.04)$ & -51 & -0.005 & $971(145)$ & $0.92(0.05)$ \\
\hline Action & 859 (147) & $0.96(0.04)$ & 851 (146) & $0.95(0.04)$ & -8 & -0.01 & $812(125)$ & $0.91(0.08)$ \\
\hline
\end{tabular}

$0.12 ; F_{2}(3,183)=9.31, p<0.001, \eta^{2}=0.07$. High-BOI words were categorized faster than low-BOI words in the entity, $t_{1}(37)=9.18, p<0.001, \eta^{2}=0.69 ; t_{2}(50.11)=4.34, p<$ $0.001, \eta^{2}=0.27$, entity-action, $t_{1}(38)=3.60, p=0.001, \eta^{2}=$ $0.25 ; t_{2}(67)=2.14, p<0.05, \eta^{2}=0.06$, and action-entity, $t_{1}(38)=2.70, p=0.01, \eta^{2}=0.16 ; t_{2}(67)=2.28, p<0.05$, $\eta^{2}=0.07$, conditions. Critically, there was no significant difference between categorization times for high- and low-BOI items in the action condition, $t_{1}<1 ; t_{2}<1$, suggesting that BOI information was only useful when participants were told that entity words would be present in the decision task ${ }^{1}$.

Results also included a significant main effect of BOI, as highBOI words were classified more quickly than low-BOI words overall, $F_{1}(1,151)=64.44, p<0.001, \eta^{2}=0.29 ; F_{2}(1,61)=$ 10.50, $p<0.01, \eta^{2}=0.14$. There was also a significant main effect of decision category, $F_{1}(3,151)=7.23, p<0.001, \eta^{2}=$ $0.13 ; F_{2}(3,183)=63.22, p<0.001, \eta^{2}=0.47$, since RTs to the critical words were fastest for the action condition $(855 \mathrm{~ms})$, followed by the entity-action condition $(939 \mathrm{~ms})$, entity condition (969 ms), and action-entity condition (1021 ms). This effect of decision category was followed up using comparisons with a Bonferroni correction. RTs in the action condition were faster than those in the entity, $t_{1}(75)=3.37, p<0.01 ; t_{2}(62)=7.14$, $p<0.01$, and action-entity conditions, $t_{1}(76)=4.72, p<0.01$; $t_{2}(66)=15.37, p<0.01$. There was no significant difference between RTs in the action and entity-action conditions in the subject analysis, $t_{1}(76)=2.29, p>0.05$, however, RTs were faster in the action than entity-action condition in the item analysis $t_{2}(66)=7.54, p<0.01$. RTs in the entity-action condition were not found to differ from RTs in the entity condition in the subject analysis, $t_{1}<1$, but entity-action RTs were significantly faster than the entity RTs in the item analysis, $t_{2}(64)=3.18$, $p<0.05$. Entity-action RTs did not differ from action-entity RTs in the subject analysis, $t_{1}(76)=2.08, p>0.05$, but entity-action RTs were significantly faster than action-entity RTs in the item analysis $t_{2}(68)=9.33, p<0.01$. Finally, RTs in the entity and action-entity conditions did not differ, $t_{1}<1 ; t_{2}(64)=2.81$, $p>0.05$.

\footnotetext{
${ }^{1}$ Although the difference is not significant, action-entity ratings were numerically lower (i.e., more entity-like) for high- versus low-BOI words. To investigate whether this difference influenced the overall pattern of results, we re-examined the data using ANCOVA analyses in which action-entity rating was a covariate. With one exception, the pattern of results revealed by these new analyses was identical to that of the reported analyses: in the original analysis of RTs in the entity-action condition, a significant BOI advantage was observed (faster RTs for high- vs. low-BOI words). This same effect was marginally significant $(p=0.062)$ in the ANCOVA analysis.
}

\section{ACCURACY ANALYSIS}

In the analysis of accuracy data, decision category and BOI were again found to interact, $F_{1}(3,151)=14.17, p<0.001, \eta^{2}=$ $0.21 ; F_{2}(3,183)=19.20, p<0.001, \eta^{2}=0.20$. There was no difference in categorization accuracy for high- versus low-BOI items in the entity-action, $t_{1}<1 ; t_{2}<1$, and action-entity conditions, $t_{1}<1 ; t_{2}<1$. In the action task, high-BOI items were categorized marginally less accurately than low-BOI words in the subject analysis, $t_{1}(38)=1.81, p=0.07, \eta^{2}=0.07$, but not the item analysis, $t_{2}(65)=1.01, p=0.31, \eta^{2}=0.01$. Finally, high-BOI words were categorized more accurately than low-BOI words in the entity condition, $t_{1}(37)=9.18, p<0.001, \eta^{2}=$ $0.69 ; t_{2}(54.04)=5.32, p<0.001, \eta^{2}=0.34$, suggesting that BOI information was most useful when participants were only told that entity words would be present in the decision task.

Results also included a main effect of BOI, as high-BOI words were categorized more accurately than low-BOI words overall, $F_{1}(1,151)=9.10, p<0.01, \eta^{2}=0.05 ; F_{2}(1,61)=3.84, p=$ $0.055, \eta^{2}=0.05$. There was also a main effect of decision category, $F_{1}(3,151)=4.86, p<0.01, \eta^{2}=0.09 ; F_{2}(3,183)=$ 17.37, $p<0.001, \eta^{2}=0.18$, with the highest accuracy rates for critical words in the action condition (0.96), followed by the action-entity condition (0.95), the entity-action condition (0.93) and the entity condition (0.91). Follow-up comparisons using the Bonferroni correction revealed that accuracy was significantly higher in the action condition than the entity condition, $t_{1}(60.50)=3.49, p<0.01 ; t_{2}(62)=3.98, p<0.01$, but not the action-entity condition, $t_{1}<1 ; t_{2}<1$. There was no significant difference between accuracy in the action versus entity-action conditions in the subject analysis, $t_{1}(59.27)=2.16, p>0.05$, however, responses were significantly more accurate in the action condition than the entity-action condition in the item analysis, $t_{2}(66)=4.15, p<0.01$. Responses were significantly more accurate in the action-entity condition than the entity condition, $t_{1}(61.47)=2.96, p<0.05 ; t_{2}(64)=3.65, p<0.01$. Accuracy was not found to differ between the action-entity and entityaction conditions in the subject analysis, $t_{1}(60.20)=1.68, p>$ 0.05 , however, responses in the action-entity condition were found to be significantly more accurate than those in the entityaction condition in the item analysis, $t_{2}(68)=3.48, p<0.01$. Finally, accuracy did not differ across the entity-action and entity conditions, $t_{1}<1$; $t_{2}(64)=1.45, p>0.05$.

\section{DISCUSSION}

The goal of the current study was to examine how task context might modulate BOI effects in lexical-semantic processing. As such, we investigated how small differences in task presentation 
(participants' advance knowledge about the types of items presented) modulated BOI effects in semantic categorization behavior. Results showed a BOI advantage, with faster classification times for high- versus low-BOI words, in the three conditions where participants were told to expect entity words. This effect was largest, and was accompanied by a significant accuracy advantage for high BOI words, when participants were only expecting entity (and non-entity) words. Critically, there was no BOI advantage for either RT or accuracy measures when participants were only expecting action (and non-action) words. Our results show three quantitatively different effects using the same set of items, indicating strong modulation of BOI effects as a function of the specific task context.

That the effect of BOI is modulated by the information participants are given about the decision category indicates a strong role for context in semantic processing and suggests that participants are able to adopt disparate task sets as a function of their expectations about what will be important in a task. Certain category labels bias participants' response behavior, perhaps by encouraging them to focus on dimensions of semantic information that are highly relevant to the decision. In the entity condition, this focus on entity-relevant dimensions magnified the BOI effect. Conversely, in the action condition, the focus on action-relevant dimensions eliminated the BOI effect. When participants were given information about both categories of words, as in the action-entity and entity-action conditions, a middle ground was reached, where it seems probable that dimensions relevant to both categories were emphasized in order to categorize stimuli. As such, our results are consistent with those of other studies showing that the type of information participants extract from word stimuli depends on the task context. For instance, Raposo et al. (2009) showed that auditory processing of action verbs was associated with motor and premotor activation when the words were presented alone or in literal sentences, but not when the words were intended figuratively, in idiomatic sentences (although cf. Boulenger et al., 2009).

The observation of BOI effects in lexical-semantic processing has been taken as evidence for the claim that bodily experience is an important aspect of semantic knowledge, activated in the process of generating meaning from print (e.g., Siakaluk et al., 2008a,b; Wellsby et al., 2011). The absence of a significant BOI effect in the "is it an action?" condition of the present study puts limits on the impact of this bodily experience dimension in semantic processing. This is not necessarily to say that some semantic processing is disembodied, but rather highlights the fact that a given embodied dimension (e.g., BOI) may only influence behavior in contexts in which it is task-relevant (Willems and Casasanto, 2011). Semantic processing in the "is it an action?"

\section{REFERENCES}

Balota, D. A., Cortese, M. J., SergentMarshall, S. D., Spieler, D. H., and Yap, M. J. (2004). Visual word recognition of single-syllable words. J. Exp. Psychol. Gen. 133, 283-316.

Balota, D. A., Yap, M. J., Cortese, M. J., Hutchison, K. I., Kessler, B.,

condition may still be grounded in sensorimotor processing, but whatever sensorimotor dimensions are most relevant to the action decision category were not captured in the present study.

We chose to examine BOI effects in the present research because BOI is a richness dimension that is relevant to a particular class of words. As such, it seemed possible that BOI might be sensitive to the kind of task framing manipulation applied here, where participants had advance knowledge that a type of word would be presented (or not). An unanswered question, however, is whether this kind of task context modulation would also be observed for other semantic richness effects. That is, are the effects of other measures of semantic richness on semantic categorization performance equally malleable? Some richness dimensions, like BOI and imageability, are derived from subjective ratings and, arguably, are thus more intuitive than other objectively-derived dimensions, like number of features, semantic neighborhood density, or contextual dispersion. That is, people can provide consistent ratings about words' BOI but our experience with the more objectively-derived number of features dimension, for instance, is that people have very little insight about whether a word has a high or low number of features. One possibility is that context effects are strongest when participants have some insight about the kinds of information associated with particular stimuli, and can use this insight to tap into the information they suspect will optimize their performance in a task. If this is the case, then the subjective semantic richness dimensions may be more malleable than the objective richness dimensions. Of course, it is also possible that this insight is not at all relevant to context modulation, and all semantic dimensions are equally context-dependent. In this latter case it should not matter whether the particular richness dimension is subjectively- or objectively-derived. These possibilities will need to be tested in future research.

The results of the present study show that even quite subtle differences in the way a task is characterized can produce substantial changes in behavioral effects, as participants made adjustments to their processing of word meaning information (based entirely on small changes in the instructions provided) in order to optimize performance in categorization tasks. As such, our results suggest that participants have strong top-down control of the semantic categorization process. This suggestion is compatible with a flexible, dynamic view of semantic processing (Kiefer and Pulvermüller, 2012).

\section{ACKNOWLEDGMENTS}

The Natural Sciences and Engineering Research Council of Canada supported this research through a Postgraduate Scholarship to CT and a Discovery Grant to Penny M. Pexman.

Barsalou, L. W. (2008). Grounded L., Simpson, G. B., and Treiman, R. (2007). The English Lexicon Project. Behav. Res. Methods 39, 445-459.

Barsalou, L. W. (1982). Contextindependent and context-dependent information in concepts. Mem. Cognit. 10, 82-93. cognition. Annu. Rev. Psychol. 59, 617-645.

Buchanan, L., Westbury, C., and Burgess, C. (2001). Characterizing semantic space: neighborhood effects in word recognition. Psychon. Bull. Rev. 8, 531-544.

Boulenger, V., Hauk, O., and Pulvermüller, F. (2009). Grasping ideas with the motor system: semantic somatotopy in idiom comprehension. Cereb. Cortex 19, 1905-1914.
Caramazza, A., and Mahon, B. Z. (2003). The organization of conceptual knowledge: the evidence from category-specific semantic 
deficits. Trends Cogn. Sci. 7, 354-361.

Collins, A. M., and Loftus, E. F. (1975). A spreading-activation theory of semantic processing. Psychol. Rev. $82,407-428$.

Davis, C. J. (2005). N-Watch: a program for deriving neighborhood size and other pshycolinguistic statistics. Behav. Res. Methods 37, 65-70.

Fodor, J. A. (1975). The Language of Thought. Cambridge, MA: Harvard University Press.

Grondin, R., Lupker, S. J., and McRae, K. (2009). Shared features dominate semantic richness effects for concrete concepts. J. Mem. Lang. 60, 1-19.

Hargreaves, I. S., White, M., Pexman, P. M., Pittman, D., and Goodyear, B. G. (2012). The question shapes the answer: the neural correlates of task differences reveal dynamic semantic processing. Brain Lang. 120, 73-78.

Hino, Y., Pexman, P. M., and Lupker, S. J. (2006). Ambiguity and relatedness effects in semantic tasks: are they due to semantic coding? J. Mem. Lang. 55, 247-273.

Hoenig, K., Sim, E-J., Bochev, V., Herrnberger, B., and Kiefer, M. (2008). Conceptual flexibility in the human brain: dynamic recruitment of semantic maps from visual, motor, and motion-related areas. J. Cogn. Neurosci. 20, 1799-1814.

Kiefer, M., and Pulvermüller, F. (2012). Conceptual representations in mind and brain: theoretical developments, current evidence and future directions. Cortex. (in press).

Kucera, H., and Francis, W. N. (1967). Computational Analysis of PresentDay American English. Providence: Brown University Press.

Pexman, P., Hargreaves, I., Siakaluk, P., Bodner, G., and Pope, J. (2008) There are many ways to be rich: effects of three measures of semantic richness on visual word recognition. Psychon. Bull. Rev. 15, 161-167.

Pexman, P. M., Holyk, G. G., and Monfils, M-H. (2003). Number-offeatures effects and semantic processing. Mem. Cognit. 31, 842-855.

Pexman, P. M., Lupker, S. J., and Hino, Y. (2002). The impact of feedback semantics in visual word recognition: number-of-features effects in lexical decision and naming tasks. Psychon. Bull. Rev. 9, 542-549.

Raposo, A., Moss, H. E., Stamatkis, E. A., and Tyler, L. K. (2009). Modulation of motor and premotor cortices by actions, action words, and action sentences. Neuropsychologia 47, 388-396.

Schneider, W., Eschman, A., and Zuccolotto, A. (2002). E-Prime Reference Guide. Sharpsburg, PA: Psychology Software Tools, Inc.
Siakaluk, P. D., Pexman, P. M., Aguilera, L., Owen, W. J., and Sears, C. R. (2008a). Evidence for the activation of sensorimotor information during visual word recognition: the bodyobject interaction effect. Cognition 106, 433-443.

Siakaluk, P. D., Pexman, P. M., Sears, C., Wilson, K., Locheed, K., and Owen, W. J. (2008b). The benefits of sensorimotor knowledge: body-object interaction facilitates semantic processing. Cogn. Sci. 32, 591-605.

Tillotson, S. M., Siakaluk, P. D., and Pexman, P. M. (2008). Body-object interaction ratings for 1,618 monosyllabic nouns. Behav. Res. Methods 40, 1075-1078.

Wellsby, M., Siakaluk, P. D., Owen, W. J., and Pexman, P. M. (2011) Embodied semantic processing: the body-object interaction effect in a non-manual task. Lang. Cogn. 3, $1-14$.

Willems, R. M., and Casasanto, D. (2011). Flexibility in embodied language understanding. Front. Psychol. 2:116. doi: 10.3389/fpsyg.2011.00116

Wilson, M. D. (1988). The MRC Psycholinguistic Database: Machine Readable Dictionary, Version 2. Behav. Res. Methods Instrum. Comput. 20, 6-11.

Yap, M. J., Tan, S. E., Pexman, P. M., and Hargreaves, I. S. (2011). Is more always better? Effects of semantic richness on lexical decision, speeded pronunciation, and semantic classification. Psychon. Bull. Rev. 18, 742-750.

Zeno, S. M., Ivens, S. H., Millard, R. T., and Duvvuri, R. (1995). The Educator's Word Frequency Guide. United States of America: Touchstone Applied Science Associates, Inc.

Conflict of Interest Statement: The authors declare that the research was conducted in the absence of any commercial or financial relationships that could be construed as a potential conflict of interest.

Received: 22 December 2011; accepted: 29 February 2012; published online: 15 March 2012.

Citation: Tousignant $C$ and Pexman PM (2012) Flexible recruitment of semantic richness: context modulates body-object interaction effects in lexical-semantic processing. Front. Hum. Neurosci. 6:53. doi: 10.3389/fnhum.2012.00053 Copyright (c) 2012 Tousignant and Pexman. This is an open-access article distributed under the terms of the Creative Commons Attribution Non Commercial License, which permits non-commercial use, distribution, and reproduction in other forums, provided the original authors and source are credited. 


\section{APPENDIX \\ ITEMS USED IN THE EXPERIMENT \\ Low-BOI words}

ash, back, band, bay, birch, brain, brass, case, flea, fog, frost, gang, hall, heart, jail, king, knight, lane, lint, lung, mink, pint, pit, prince, roof, song, spade, star, stripe, sun, tail, tar, tomb, well, zoo

\section{High-BOI words}

belt, boot, bowl, cage, cart, child, couch, feet, food, friend, gate, gift, ham, hat, ice, man, mat, mole, nail, neck, palm, pearl, pie, priest, purse, room, seat, silk, stair, string, suit, toy, tube, vest, wheel

\section{Action words}

act, assist, attend, beg, blow, build, bury, carry, choose, come, cope, draw, earn, eat, escape, fight, gasp, give, glare, hear, hide, hold, hunt, ignore, jump, kick, kill, kiss, lean, learn, lift, make, manage, meet, move, nod, offend, pat, play, pour, punish, read, rise, rob, save, scream, see, seek, sell, sew, shiver, shout, sigh, sing, sit, sleep, smash, smile, stand, stop, stride, sue, swim, talk, teach, tell, think, vote, walk, wear 